\title{
Effect of green tea catechins in patients with high-grade prostatic intraepithelial neoplasia: Results of a short-term double-blind placebo controlled phase II clinical trial
}

\author{
Salvatore Micali ${ }^{1}$, Angelo Territo ${ }^{1}$, Giacomo Maria Pirola ${ }^{1}$, Nancy Ferrari ${ }^{1}$, Maria Chiara Sighinolfi ${ }^{1}$, \\ Eugenio Martorana $^{1}$, Michele Navarra ${ }^{2}$, Giampaolo Bianchi ${ }^{1}$ \\ ${ }^{1}$ Department of Urology, Bagiovara Hospital, University of Modena and Reggio Emilia, Italy; \\ 2 Department of Chemical, Biological, Pharmaceutical and Environmental Sciences, University of Messina, Italy
}

\begin{abstract}
Summary Background and study objective: Several studies suggest a protective role of green tea catechins against prostate cancer (PCa). In order to evaluate the efficacy of green tea catechins for chemoprevention of $P C a$ in patients with high-grade prostate intraepithelial neoplasia (HG-PIN) we performed a phase II clinical trial. Methods: Sixty volunteers with HG-PIN were enrolled to carry out a double-blind randomized placebo-controlled phase II clinical trial. Treated group took daily $600 \mathrm{mg}$ of green tea catechins (Categ Plus ${ }^{\circledR}$ ) for 1 year. Patients were screened at 6 and 12 months through prostatic biopsy and measurements of prostate-specific antigen (PSA).

Results: Despite the statistically significant reduction of PSA observed in subjects who received green tea catechins for 6 and 12 months, we did not find any statistical difference in PCa incidence between the experimental groups neither after 6 nor after 12 months. However, throughout the one-year follow-up we observed very limited adverse effects induced by green tea catechins and a not significant improvement in lower urinary tract symptoms and quality of life.

Conclusions: Although the small number of patients enrolled in our study and the relatively short duration of intervention, our findings seems to deny the efficacy of green tea catechins.

However, results of our clinical study, mainly for its low statistical strength, suggest that the effectiveness of green tea catechins should be evaluated in both a larger cohort of men and longer trial.
\end{abstract}

KEY WORDS: Prostate cancer; Green tea catechins; High-grade intra-epithelial neoplasia (HG-PIN); Herbal medicines; PSA; Complementary therapies.

Submitted 9 June 2017; Accepted 3 August 2017

\section{INTRODUCTION}

Prostate cancer (PCa) is a leading cause of cancer related death among men in Western countries, representing a major public health problem with high economic and social costs. However, it is typically diagnosed in men over 50 years of age and, as clinically significant PCa,

\footnotetext{
Funding and competing interests: This clinical trial received no specific funding. The authors declare that there is no conflict of interest regarding the publication of this paper. Sofar S.P.A, Milan provided free samples of Categ Plus ${ }^{\circledR}$ employed in this study, without any role in data collection and analysis, decision to publish, or preparation of the paper.
}

usually, requires more than two decades for its development. Thus, it can be considered as an important chance for early prediction or chemoprevention through therapeutic or nutritional interventions, especially in the case of pre-malignant lesions such as high-grade prostate intraepithelial neoplasia (HG-PIN) (1). A chemoprevention approach in patients with high risk of $\mathrm{PCa}$ can be proposed in order to reduce the disease progression rate. It is known that natural product can be used to prevent and/or alleviate several health disorders (2-4), including those affecting the urinary tract (5). Moreover, regular dietary habits coupled with a healthy lifestyle may protect against certain types of cancer $(6,7)$, including PCa. In particular, epidemiological studies have shown that Asiatic populations have lower rates of PCa compared to others races and that Asian men who adopt a lifestyle typical of Western countries because of migration to the United States, have a significantly higher risk of PCa when compared to their native Asian counterparts (8). These findings strengthen the hypothesis that environmental factors may contribute to PCa development and that the use of dietary agents such as green tea may be an important way to prevent or slow down the process of carcinogenesis, hence the growing interest toward both dietary supplements and complementary and alternative medicine. In such context, diet-derived polyphenols have received great attention among nutritionists, food scientists, and consumers for their health-promoting effects, including their use in the chemoprevention of PCa (9), because oxidative stress has been implicated in the aetiopathogenesis of PCa (10). Regardless, unresolved issues still linger.

Tea is the most consumed beverage worldwide, second only to water in terms of popularity. It is prepared by infusion of the Camelia sinensis L. (Theaceae) leaves.

On the basis of both the differences in the processing methods and chemical composition, it could be classified in three main types, the green tea (unfermented), the oolong tea (partially fermented) and the black tea (fully fermented). Habitual green tea consumption has long been associated with preventive effects against chronic pathologies including both heart and neurodegenerative disease and cancer. In the latter field, several in vitro and 
in vivo studies showed that tea and its bioactive molecules might modify the incidence and the progression of PCa through their antioxidant properties and the ability to interact with specific intracellular targets in cancerous cells (11).

The beneficial effects of green tea are attributed especially to its water soluble polyphenolic flavonoids, known as catechins, including epicatechin (EC), epigallocatechin (EGC), epicatechin-3-gallate (ECG) and the major flavonoid (-)-epigallocatechin-3-gallate (EGCG).

Their content is $30-40 \%$ of dry green tea weight, and EGCG may represent up to $50 \%$ of the catechins by weight. However, other polyphenols present in green tea are quercetin, myricitrin and kaempferol.

Bettuzzi et al. (12) proposed the green tea catechins as chemopreventive agents against prostate cancer in men with HG-PIN, confirming this suggestion in a long term follow up study (13). However, others studies (14) often report conflicting results, suggesting that habitual green tea consumption may not provide the alleged protection against cancer. Therefore, the aim of the study was to evaluate the efficacy of green tea catechins for chemoprevention of PCa in patients with HG-PIN by a double-blind randomized placebo-controlled phase II clinical trial.

\section{Methods}

\section{Study design}

Sixty volunteers with HG-PIN were enrolled to carry out a double-blind randomized placebo-controlled phase II clinical trial in order to investigate whether green tea catechins could prevent the occurrence of PCa in men at high-risk.

\section{Ethics approval and consent to participate}

This study was approved by the Modena Ethic Committee (Italy) on 2007-04-10 (number 23/07) and carried out in the Modena University Hospital (Italy) from May 2007 to February 2011. The recommendations of the Declaration of Helsinki and the guidelines of the International Conference on Harmonization Good Clinical Practice were observed. All volunteers have been properly informed about the clinical trial and signed a free informed consent form.

Study objectives. The primary objective was to determine the efficacy of green tea catechins for chemoprevention of PCa in patients with HG-PIN. Therefore, the first goal of our study was to point out PCa incidence during the 1-year study in the two arms. Moreover, during the whole study, we recorded possible changes in total serum prostate-specific antigen (PSA) values (secondary objective), together with the toxicity evaluation as well as the patient's lower urinary tract symptoms (LUTS) and quality of life (QoL) scores assessments by questionnaires. Subjects who were detected PCa during the clinical trial were excluded from the study and subjected to chemotherapy.

Study population. Eligibility criteria for enrolment included: Caucasian men aged between 55 to 65 years old with HG-PIN, as assessed by prostate biopsy. All volunteers were properly advised about the clinical trial and signed a free informed consent form. Exclusion criteria included: men aged $>65$ years, previous malignancies, cancer diagnosis, antiandrogenic or chemoprevention therapies, obesity, diabetes or other endocrinologic diseases. The subjects enrolled in the trial were instructed to abstain from green tea and its derivatives, antioxidants or nutritional supplements and herbal therapies.

\section{Study procedures}

During the initial visit, volunteers underwent an interview to obtain medical history as well as a brief physical assessment including a digital rectal examination. Moreover, blood samples were collected for complete blood count and serum PSA levels. Finally, baseline assessments of LUTS and QoL, were obtained.

Upon determination of eligibility, a total of 60 patients participating to the clinical trial were randomized (1:1) to receive daily green tea catechins $600 \mathrm{mg}$ ( 2 tablets of 300 $m g$ of Categ Plus ${ }^{\circledR}$, Sofar S.P.A, Milan, Italy) or placebo for 1 year. The random allocation sequence was obtained by means of "Easy Random Picker" software (TrustFm ${ }^{\odot} 1998$ 2016). Both participants and care providers were blinded after assignment to interventions, in order to avoid any bias. Two capsules of Categ Plus ${ }^{\circledR}$ or placebo per day were given to all subjects by the clinical trial investigators, according to the double blind method.

Follow up was carried out at 6 and 12 months with toxicity assessment (side effects), LUTS and QoL evaluation, medical examination, rectal inspection, serum PSA levels and prostate biopsy. PSA measurements as well as immunohistochemistry evaluation of prostatic biopsies were performed in the same hospital's central laboratory where was carried out the clinical trial (Modena University Hospital, Italy). Possible changes in LUTS, using the International Prostate Symptom Score (IPSS), and QoL were evaluated as described $(15,16)$.

\section{Statistical analysis}

Statistical analyses of results were performed using the Student's t test. P-values $<0.05$ were considered statistically significant.

\section{RESULTS}

First, we have ensured that the randomization of volunteers in the two arms of the clinical trial had resulted in two homogeneous groups with one another. A Consort flow diagram of the study is shown in Figure 1. In particular, at the time of enrollment the age of subject, their weight, the PSA value and the prostate volume were recorded and statistically analyzed. In Table 1 are reported their mean values. None of the variables considered were significantly different in the two arms (age, $p=0.8$; PSA value, $\mathrm{p}=0.16$; prostate volume, $\mathrm{p}=0.23$ ). The two groups were statistically analyzed to ensure homogeneity between the two groups by the $t$ test of Student (age, $\mathrm{p}=$ 0.580 ; PSA value, $\mathrm{p}=0.790$; prostate volume, $\mathrm{p}=0.738$ ). Among the 60 patients that entered the study, 44 subjects completed the study (22 cases and 22 controls) with a drop out of $26.7 \%$, whereas minimal side effects were recorded. Indeed, although the majority of patients tolerated the green tea relatively well, 8 patients with- 
Figure 1.

Consort flow diagram.

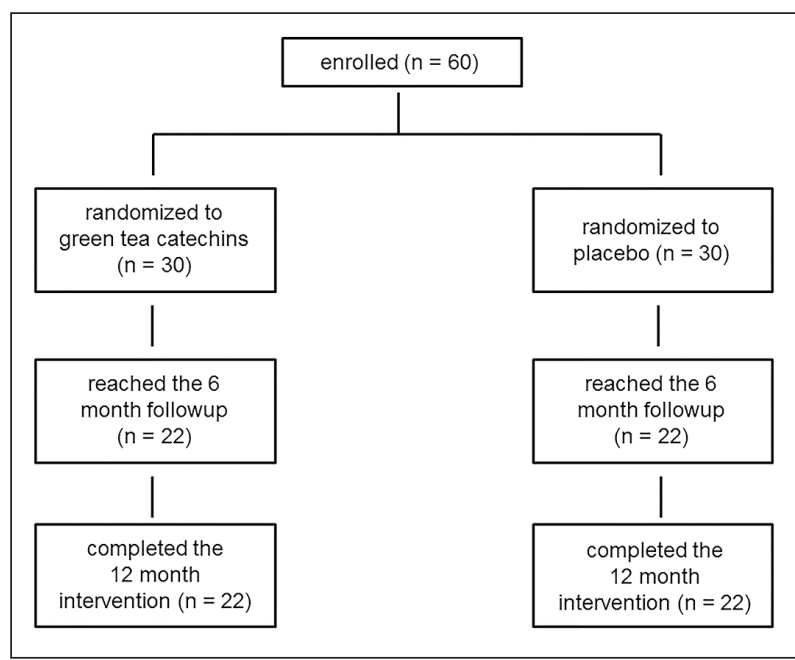

Table 1.

Patient characteristics at the time of enrollment (mean $\pm \mathrm{SD}$ ).

\begin{tabular}{|l|c|c|c|c|}
\hline & $\begin{array}{c}\text { Mean } \\
\text { age }\end{array}$ & $\begin{array}{c}\text { Weight } \\
\text { (kg) }\end{array}$ & $\begin{array}{c}\text { Prostate } \\
\text { volume (ml) }\end{array}$ & $\begin{array}{c}\text { PSA } \\
\text { value }\end{array}$ \\
\hline $\begin{array}{l}\text { Total population } \\
\text { enrolled }\end{array}$ & $64.34 \pm 8.4$ & $82.77 \pm 6.6$ & $44.74 \pm 16$ & $5.31 \pm 2.5$ \\
\hline $\begin{array}{l}\text { Placebo arm } \\
\begin{array}{l}\text { Green tea } \\
\text { catechins- } \\
\text { treated arm }\end{array}\end{array}$ & $64.4 \pm 8.9$ & $80.9 \pm 6.4$ & $42.15 \pm 15$ & $4.7 \pm 2.5$ \\
\hline
\end{tabular}

drew from the clinical study because one selves' decision. When toxicity did occur, it was of Grade 1 or 2, including the following symptoms: nausea, emesis, abdominal pain, insomnia, fatigue and diarrhea.

Figure $2 \mathrm{~A}$ shows that the incidence of new diagnoses of $\mathrm{PCa}$ at 6 months was 9\% (2/22) in the green tea catechins-treated arm, and $18.1 \%(4 / 22)$ in the placebo group; although these values are the one of the other half, the difference between green tea-treated and placebo groups has not reached the statistical significance $(\mathrm{p}=0.5)$. Moreover, at 12 months follow up, two more cases of $\mathrm{PCa}$ were diagnosed in the green tea catechins group, reaching a 18\% of $\mathrm{PCa}$ incidence also in the treated arm (Figure 2B).

In the green tea catechins group we observed a progressive reduction of the PSA value throughout the 1-year study, as well as we found a deceleration of the PSA rise in the green tea catechins group with respect to the placebo ones, however without reaching statistical significance (Table 2). Interestingly, after 12 months of treatment we found a significant reduction of PSA values in the green tea catechins group with respect to the placebo ones $(3.85 \pm 1.8 \mathrm{ng} / \mathrm{dl}$ and $5.83 \pm 2.65$, respectively; $\mathrm{p}<0.05$; Table 2).

Finally, a certain improvement in LUTS and QoL scores was found by analyzing the patient's questionnaires filled by the subjects belonging to green tea catechins group throughout the 1-year study. However, no significant differences between the treatment and placebo arms were observed from baseline to end of study (data not shown).

Figure 2.

Incidence of new diagnosis of PCa at 6 (A) and 12 (B) months' follow up.

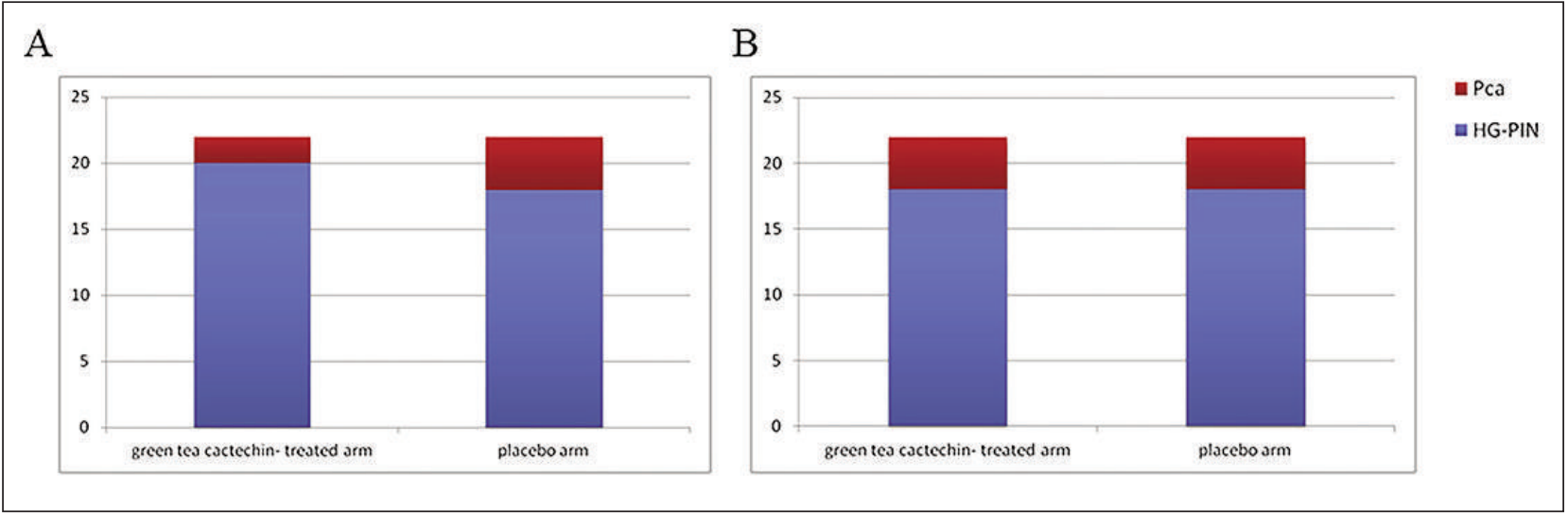

Table 2.

PSA values $(\mathrm{ng} / \mathrm{ml})$ throughout the 1-year study (mean $\pm S D$ ).

\begin{tabular}{|l|c|c|c|}
\hline & $\begin{array}{c}\text { Green tea } \\
\text { catechins-treated arm }\end{array}$ & $\begin{array}{c}\text { Placebo } \\
\text { group }\end{array}$ & $\begin{array}{c}\text { P } \\
\text { value }\end{array}$ \\
\hline Time of enrolment & $5.9 \pm 2.3$ & $4.7 \pm 2.5$ & \\
\hline $\mathbf{6}$ months & $4.9 \pm 1.9$ & $5.2 \pm 2.1$ & N.S. \\
\hline $\mathbf{1 2}$ months & $3.8 \pm 1.8$ & $5.8 \pm 2.6$ & $<0.05$ \\
\hline
\end{tabular}

\section{Discussion}

PCa is considered the most common malignancy and the second leading cause of cancer death among men in United States and Europe. During its progression, cell phenotype changes from normal to severe dysplasia (high-grade prostatic intraepithelial neoplasia), to early (superficial) cancers, and finally to metastatic disease. The occurrence of latent PCa is consistently distributed, suggesting that external issues such as diet, physical 
activity, and other lifestyle factors may be important in the evolution into aggressive clinical diseases (17).

HG-PIN is a premalignant condition, defined by neoplastic development of epithelial cells among pre-existing benign prostatic acini or ducts, appearing as a sort of intermediate stage between benign epithelium and malignant carcinoma. Histologically, the main difference with the prostate carcinoma is the conservation of the basement membrane, which orients pathologists in indicating an HG-PIN. On the other hand, cellular parameters can be mismatched with a carcinoma, like the prominent and abnormal nuclei, that are also characteristic in HG-PIN (18). The incidence of HG-PIN on prostatic biopsy averages approximately 15\%, increasing with patient's age. Moreover, some areas of HG-PIN are frequently found around prostatic cancerous lesions and evolution into carcinoma is estimated in about 30\% of cases (19). Recent literature states that 30\% of men with HG-PIN would develop prostate cancer within 1 year after repeated biopsy (1). In this contest, the chemoprevention (the administration of agents to prevent the induction or to delay the progression of cancers) play an important role, trying to stop multistage carcinogenesis before its development in malignancy. PCa with its high prevalence and long latency provides a promising approach for evaluating agents for chemoprevention.

It is known that the possible protective action of green tea on $\mathrm{PCa}$ is due to the presence of catechins, above all EGCG, since black tea, poor of these compounds, does not play a role in prevention from PCa (20). An important clinical trial investigating the effectiveness of green tea catechins on PCa was carried out by Bettuzzi et al. (12), suggesting that green tea polyphenols may be effective for treating premalignant lesions before PCa develops. In this prospective, randomized, double-blind, placebo-controlled clinical trial, 60 volunteers with HGPIN received orally $600 \mathrm{mg}$ per day of green tea catechins or placebo for one year, and were subjected to medical examinations and prostate biopsies at 6 at 12 months. Authors concluded that green tea catechins might provide a $90 \%$ of chemoprevention efficacy. Following the same HG-PIN cohort of subject for two years (despite a considerable dropout rate), the reduction of PCa incidence in the green tea catechins arm still remained significant, thus suggesting a long lasting inhibition of cancer development achieved with one-year therapy (13).

In our study, we failed to confirm a clear association between green tea catechins and PCa chemoprevention, suggesting that the lack of effectiveness we described could be due to the somewhat small number of enrolled volunteers as well as to limited length of the study. However, results of our clinical trial are consistent with studies supporting the notion that green tea intake does not protect against prostate cancer. For instance, a prospective cohort study performed using data from a follow-up database of 27.293 of Singapore Chinese men, concluded that there was no association between daily green tea intake and prostate cancer risk, compared with no green tea intake (21). Moreover, results of a prospective cohort study that involved 19.561 Japanese men from Ohsaki to whom was self-administered a question- naire suggested that green-tea intake did not reduce PCa (22). On the contrary, a case-control study in China found that green-tea intake was associated with a lower risk of prostate cancer (23), whereas a case-control study in Japan showed a modest reduction in PCa (24).

Borderline results were obtained also in a randomized, double-blind, placebo-controlled trial performed in men with PCa scheduled to undergo radical prostatectomy that received daily either a drug containing $800 \mathrm{mg}$ green tea catechins or placebo for 3 to 6 weeks before surgery. Results of the study indicate that the treatment with green tea catechins resulted in favorable but not statistically significant changes in serum PSA as well as the proportion of subjects who had a decrease in Gleason score between biopsy and surgical specimens (25). A phase II trial aimed to estimate percentage of patients with androgen independent metastatic PCa who sustained a decline in PSA level with green tea, showed its limited capability to breakdown PSA, although no patient manifested a tumor response during the months of treatment (26). Another prospective clinical trial for hormone refractory prostate cancer indicated minimal clinical activity of green tea extract capsules in 15 men (27). Finally, a meta-analysis indicated that consumption of green tea may have a protective effect on PCa in Asian populations, suggesting that further prospective cohort studies are needed to obtain a definitive conclusion in this field, especially with regard to the protective role of green tea on PCa across different regions apart from Asia (20).

It is known that inflammation plays an important role in the aetiology of prostate cancer (28). On the other hand, several in vitro and in vivo studies have shown that both green tea and their catechins may inhibit carcinogenesis during the initiation, promotion and progression stages through many mechanisms, including the antioxidant effects (29). Moreover, Mukherjee et al. (30) reported that EGCG suppresses inflammation in prostate cancer cells, thus reducing the risk of PCa. Therefore, we speculate that the significant reduction of PSA values throughout the one-year study, as well as the not significant decrease of PCa at the 6 month follow up, we observed in our study, could be linked to the anti-inflammatory property of green tea. This hypothesis was also supported by a recent open label, phase II clinical trial carried out in 113 men diagnosed with prostate cancer randomized who received six cups daily of brewed green tea or brown tea or water for three to eight weeks prior to radical prostatectomy (31). In prostate tissue, Henning et al. found that green tea consumption led to a significant decrease in nuclear immunostaining of nuclear factor kappa B (NFKB) compared to water control, which may reduce inflammatory processes that may contribute to prostate cancerogenesis. Moreover, evidences of both systemic antioxidant effect (reduced urinary 8-hydroxydeoxyguanosine) and small but statistically significant decrease in PSA levels were observed. LUTS are a complex of obstructive (intermittency flow issue, incomplete voiding, weak urinary stream) and irritative (pollakiuria nocturia, urgency and burning during urination) symptoms that affect the quality of life and are quite common in men over the age of 60 years and in those with benign prostatic hyperplasia (32). Interestingly, the analysis of 
questionnaires administered to subjects participating in the clinical study showed a small but not significant improvement of LUTS or QoL scores in the green teatreated group respect to the control one. This finding supports the hypothesis that the anti-inflammatory effect of green tea catechins both can determine the reduction in blood levels of PSA and can relieve the symptoms of LUTS, thus enhancing the quality of life.

\section{Conclusions}

In recent decades, natural drugs gained particular attention mainly because of their antioxidant and anti-inflammatory activities exploitable in the prevention of certain chronic degenerative pathologies (4, 33-35). However, the increased employment of alternative and complementary medicines requires a lot of attention from the scientific community in order to evaluate the effectiveness and safety of herbal preparations since studies have cast some doubts on whether their use is really producing an alternative to synthetic drugs $(5,36,37)$.

Although numerous in vitro and in vivo studies have suggested a protective effect of green-tea polyphenols against development of prostate cancer, to date this has not been unequivocally demonstrated in humans. Despite the small number of patients we enrolled and the relatively short duration of intervention, results of our clinical trial indicate that green tea catechins do not reduce the chance of a later development of PCa in men with HGPIN. However, the minor incidence of new diagnoses of PCa at 6 months follow-up (albeit not statistically significant) and the significant reduction of PSA levels at both 6 and 12 months follow-up, associated to the quite favourable trend that we observed in our clinical trial (very limited adverse effects and improvement in LUTS and quality of life) suggest that future studies with a larger cohort of men and longer durations of interventions are warranted. Moreover, the results of our trial provide a glimpse of the possibility that the green tea catechins might be useful, alone or in combination with other drugs, for the prevention or treatment of other prostatic pathologies.

\section{ETHICS APPROVAL AND CONSENT TO PARTICIPATE}

This study was approved by the Modena Ethic Committee (Italy) on 2007-04-10 (number 23/07) and carried out in the Modena University Hospital (Italy) from May 2007 to February 2011. All volunteers have been properly informed about the clinical trial and signed a free informed consent form.

\section{REFERENCES}

1. Bostwick DG, Liu L, Brawer MK, Qian J. High-grade prostatic intraepithelial neoplasia. Rev Urol. 2004; 6:171-179.

2. Paterniti I, Cordaro M, Campolo M, et al. Neuroprotection by association of palmitoylethanolamide with luteolin in experimental Alzheimer's disease models: the control of neuroinflammation. CNS Neurol Disord Drug Targets. 2014; 13:1530-41.

3. Filocamo A, Bisignano C, Ferlazzo N, et al. In vitro effect of berg- amot (Citrus bergamia) juice against cagA-positive and-negative clinical isolates of Helicobacter pylori. BMC Complement Altern Me. 2015; 15:256.

4. Marino A, Paterniti I, Cordaro M, et al. Role of natural antioxidants and potential use of bergamot in treating rheumatoid arthritis. PharmaNutrition. 2015; 3:53-59.

5. Micali S, Isgro G, Bianchi G, et al. Cranberry and recurrent cystitis: more than marketing? Crit Rev Food Sci Nutr. 2014; 54:1063-75.

6. Ferlazzo N, Cirmi S, Russo M, et al. NF-KB mediates the antiproliferative and proapoptotic effects of bergamot juice in Hep G2 cells. Life Sci. 2016; 146:81-91.

7. Navarra M, Ferlazzo N, Cirmi S, et al. Effects of bergamot essential oil and its extractive fractions on SH-SY5Y human neuroblastoma cell growth. J Pharm Pharmacol. 2015; 67:1042-53.

8. Lee J, Demissie K, Lu SE, Rhoads GG. Cancer incidence among Korean-American immigrants in the United States and native Koreans in South Korea. Cancer Control. 2007; 14:78-85.

9. Khan N, Syed DN, Ahmad N, et al. A dietary antioxidant for health promotion. Antioxid Redox Signal. 2013; 19:151-162.

10. Minciullo PL, Inferrera A, Navarra M, et al. Oxidative stress in benign prostatic hyperplasia: a systematic review. Urol Int. 2015; 94:249-54.

11. Johnson JJ, Bailey HH, Mukhtar H. Green tea polyphenols for prostate cancer chemoprevention: a translational perspective. Phytomedicine. 2010; 17:3-13.

12. Bettuzzi S, Brausi M, Rizzi F, et al. Chemoprevention of human prostate cancer by oral administration of green tea catechins in volunteers with high-grade prostate intraepithelial neoplasia: a preliminary report from a one-year proof-of-principle study. Cancer Research. 2006; 66:1234-40.

13. Brausi M, Rizzi F, Bettuzzi S. Chmorevention of human prostate cancer by green tea catechins: two years later. A follow up update. Eur Urol. 2008; 54:472-473.

14. Jacob SA, Khan TM, Lee LH. The Effect of Green Tea Consumption on Prostate Cancer Risk and Progression: A Systematic Review. Nutr Cancer. 2017; 69:353-364.

15. Denis LJ. Future implications for the management of benign prostatic hyperplasia. Eur Urol. 1994; 25:29-34.

16. Grumann M, Schlag PM. Assessment of quality of life in cancer patients: complexity, criticism, challenges. Onkologie. 2001; 24:10-5.

17. Villeneuve PJ, Johnson KC, Kreiger N, Mao Y. Risk factors for prostate cancer: results from the Canadian National Enhanced Cancer Surveillance System. Cancer Cause Control. 1999; 10:355367.

18. Kim HL, Yang XJ. Prevalence of high-grade prostatic intraepithelial neoplasia and its relationship to serum prostate specific antigen. Int Braz J Urol 2002; 28:413-417

19. Bostwick DG, Qian J. Atypical adenomatous hyperplasia pf the prostate. Relationship with carcinoma in 217 whole-mount radical prostatectomies. Am J Surg Pathol 1995; 19:506-518.

20. Zheng J, Yang B, Huang T, et al. Green tea and black tea consumption and prostate cancer risk: an exploratory meta-analysis of observational studies. Nutr Cancer. 2011; 63:663-72.

21. Montague JA, Butler LM, Wu AH, et al. Green and black tea intake in relation to prostate cancer risk among Singapore Chinese. Cancer Causes Control. 2012; 23:1635-41.

22. Kikuchi N, Ohmori K, Shimazu T, et al. No association between 
green tea and prostate cancer risk in Japanese men: the Ohsaki Cohort Study. Br J Cancer. 2006; 95:371-373.

23. Jian L, Xie LP, Lee AH, Binns CW. Protective effect of green tea against prostate cancer: a case-control study in southeast China. Int J Cancer. 2004; 108:130-135.

24. Sonoda T, Nagata Y, Mori M, et al. A case-control study of diet and prostate cancer in Japan: possible protective effect of traditional Japanese diet. Cancer Sci. 2004; 95:238-42.

25. Nguyen MM, Ahmann FR, Nagle RB, et al. Randomized, double bind, placebo controlled trial of Polyphenon E in prostate cancer patients before prostatectomy: evaluation of potential chemopreventive activities. Cancer Prev Res. 2012; 5:190-298.

26. Jatoi A, Ellison N, Burch PA, et al. A phase II trial of green tea in the treatment of patiens with androgen independent metastatic prostate carcinoma. Cancer. 2003; 97:1142-6.

27. Choan E, Segal R, Jonker D, et al. A prospective clinical trial of green tea for hormone refractory prostate cancer: an evaluation of the complementary/alternative therapy approach. Urol Oncol. 2005; 23:108-113.

28. Yli-Hemminki TH, Laurila M, Auvinen A, et al. Histological inflammation and risk of subsequent prostate cancer among men with initially elevated serum prostate-specific antigen (PSA) concentration in the Finnish prostate cancer screening trial. BJU Int. 2013; 112:735-41.

29. Lambert JD, Elias RJ. The antioxidant and pro-oxidant activities of green tea polyphenols: a role in cancer prevention. Arch Biochem Biophys. 2010; 501:65-72.
30. Mukherjee S, Siddiqui MA, Dayal S, et al. Epigallocatechin-3gallate suppresses proinflammatory cytokines and chemokines induced by Toll-like receptor 9 agonists in prostate cancer cells. J Inflamm Res. 2014; 7:89-101.

31. Henning SM, Wang P, Said JW, et al. Randomized clinical trial of brewed green and black tea in men with prostate cancer prior to prostatectomy. The Prostate. 2015; 75:550-559.

32. Marberger M. Medical management of lower urinary tract symptoms in men with benign prostatic enlargement. Adv Ther. 2013; 30:309-19.

33. Ferlazzo N, Cirmi S, Calapai G, et al. Anti-inflammatory activity of Citrus bergamia derivatives: where do we stand? Molecules. 2016; $21: 1273$.

34. Ferlazzo N, Visalli G, Cirmi S, et al. Natural iron chelators: protective role in A549 cells of flavonoids-rich extracts of Citrus juices in $\mathrm{Fe}(3+)$-induced oxidative stress. Environ Toxicol Pharmacol. 2016; 43:248-56.

35. Currò M, Risitano R, Ferlazzo N, et al. Citrus bergamia juice extract attenuates $\beta$-amyloid-induced pro-inflammatory activation of THP-1 cells through MAPK and AP-1 pathways. Sci Rep. 2016; 6:20809

36. Cirmi S, Ferlazzo N, Lombardo GE, et al. Chemopreventive agents and inhibitors of cancer hallmarks: may Citrus offer new perspectives? Nutrients. 2016; 8:698.

37. Cirmi S, Ferlazzo N, Lombardo GE, et al. Neurodegenerative diseases: might Citrus flavonoids play a protective role? Molecules. 2016; 21:1312.

\section{Correspondence}

Salvatore Micali, MD

salvatore.micali@unimore.it

Territo Angelo, MD

territoangelo@tiscali.it

Pirola Giacomo Maria, MD

gmo.pirola@gmail.com

Ferrari Nancy, MD

nancyferrari@virgilio.it

Sighinolfi Maria Chiara, MD

sighinolfic@yahoo.com

Martorana Eugenio, MD

eugeniomartorana@libero.it

Bianchi Giampaolo, MD

bianchi.giampaolo@unimore.it

Bagiovara Hospital, University of Modena and Reggio Emilia

Via Pietro Giardini, 1355, Modena, I-41126, Italy

Michele Navarra, MD (Corresponding Author)

mnavarra@unime.it

Department of Chemical, Biological, Pharmaceutical and Environmental

Sciences, University of Messina

Viale Annunziata, I-98168, Messina, Italy 